\title{
Intraocular Hemorrhage After Intra-Arterial Chemotherapy for Retinoblastoma in Sickle Cell Trait
}

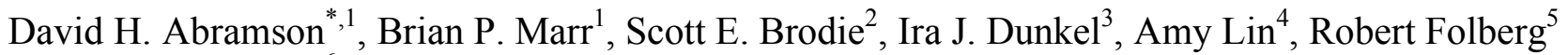 \\ and Y. Pierre Gobin ${ }^{6}$
}

\author{
${ }^{I}$ Ophthalmic Oncology Service, Memorial Sloan-Kettering Cancer Center, New York, USA \\ ${ }^{2}$ Department of Ophthalmology, Mount. Sinai School of Medicine, New York, USA \\ ${ }^{3}$ Department of Pediatrics, Memorial Sloan-Kettering Cancer Center, New York, USA \\ ${ }^{4}$ Department of Pathology, University of Illinois, Chicago, IL, USA \\ ${ }^{5}$ Departments of Biomedical Sciences, Ophthalmology, and Pathology, Oakland University William Beaumont \\ School of Medicine, Rochester, MI-, USA \\ ${ }^{6}$ Interventional Neuroradiology Service, Departments of Neurosurgery, Neurology and Radiology, Weill Cornell \\ Medical College, New York, USA
}

\begin{abstract}
Massive intraocular hemorrhage developed in a child with advanced unilateral retinoblastoma after intrarterial treatment with Melphalan and Topotecan. The child tested positive for sickle cell trait. Sickle cell trait may predispose such children to slower vascular transit time, hypoxia, sickling and vascular occlusion caused by catheter induced decreased flow. Enucleation confirmed the ultrasound and selective angiogram findings in addition to a completely calcified tumor. Clinicians should be on the lookout for the association of sickle-cell disease/trait and intraocular hemorrhages after intraarterial chemotherapy to fully understand its clinical significance.
\end{abstract}

Keywords: Retinoblastoma, sickle cell, intra-arterial chemotherapy, enucleation.

\section{INTRODUCTION}

Sickle cell trait is associated with occlusive vascular disease in adults and children under circumstances of dehydration and lowered oxygen tension (such as altitude, unpressurized aircraft) including simple dehydration and following vigorous exercise [1]. We recently treated a young child with sickle cell trait and advanced unilateral non familial retinoblastoma who developed massive subretinal hemorrhage. The enucleated eye showed calcified tumor and massive hemorrhage, possibly a consequence of sickling due to lowered oxygen tension related to slowing of blood flow during treatment.

\section{REPORT OF A CASE}

A one year old African American baby was referred for leukocoria in the left eye. That eye had no response to light, an afferent pupillary defect but normal motility and anterior segment. The fellow eye had normal fixation and following behavior and a normal ophthalmic exam. The involved eye had a total retinal detachment with subretinal seeding (Fig. 1a) and vitreous seeds confirmed on ultrasound (Fig. 1b) and Magnetic Resonance Imaging. The electroretinogram was extinguished in the involved eye, and normal in the fellow

*Address correspondence to this author at the Ophthalmic Oncology Service, Memorial Sloan-Kettering Cancer Center, 70 East 66th Street, New York, NY 10021, USA; Tel: 212-744-1700; Fax: 212-734-2553;

E-mail: abramsod@mskcc.or

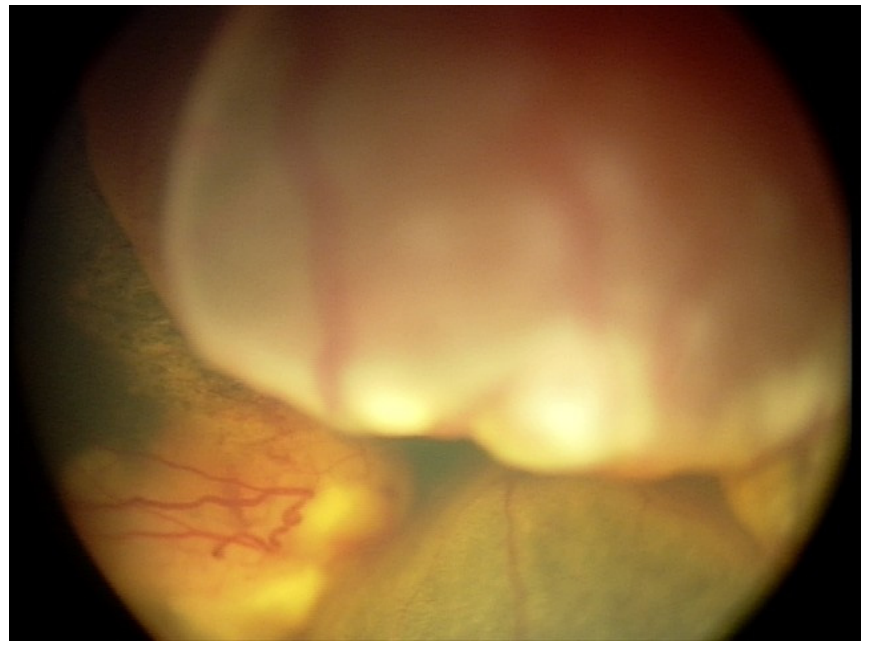

Fig. (1a). Fundus Photograph at diagnosis demonstrating total retinal detachment and subretinal tumor.

eye. Superselective delivery of chemotherapy (Melphalan 4 $\mathrm{mg}$ and Topotecan $0.3 \mathrm{mg}$ ) via the ophthalmic artery was performed and a good choroidal blush was seen (Fig. 1c). One month later an exam under anesthesia revealed a recent vitreous hemorrhage but reduced tumor volume and diminished retinal detachment, so a second treatment was performed. During the second treatment selective ophthalmic artery angiography showed that the ophthalmic artery filled well with contrast dye but the choroidal blush had 


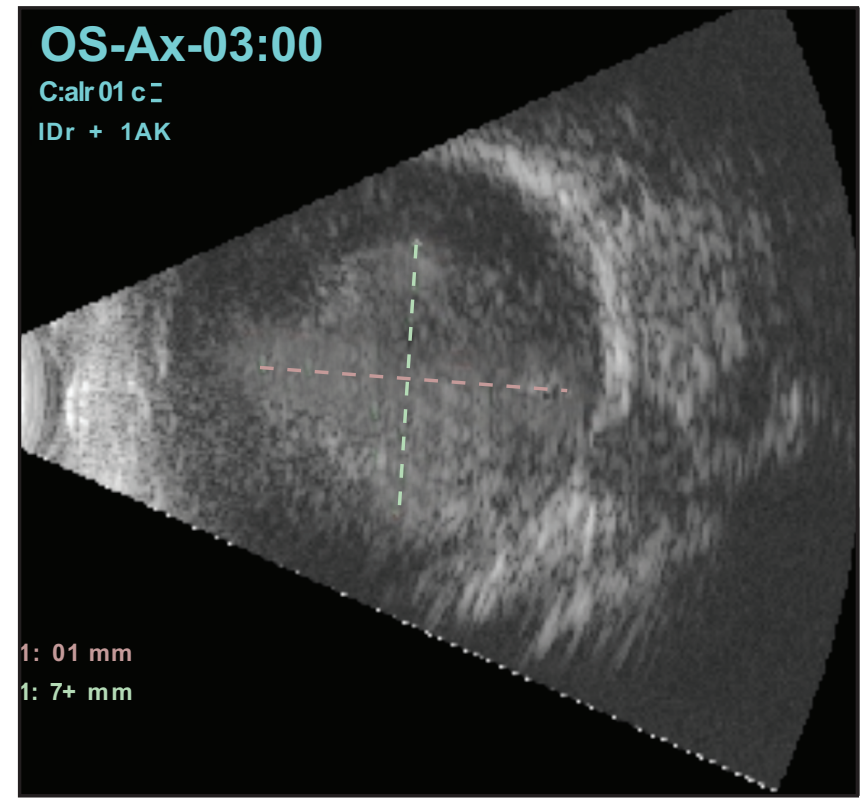

Fig. (1b). B-Scan ultrasound at diagnosis showing massive intraocular tumor filling most of the vitreous

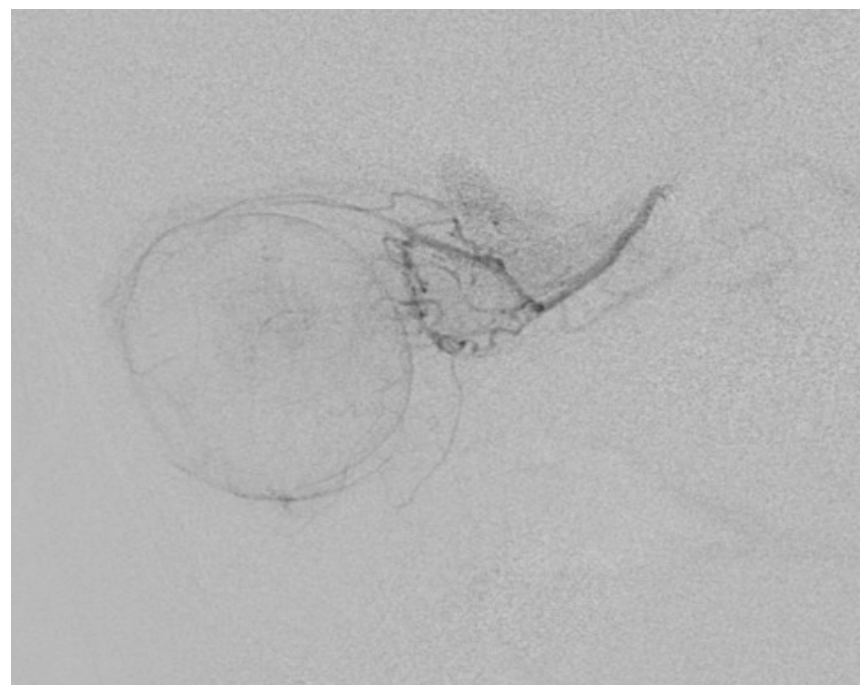

Fig. (1c). Arteriogram during first treatment showing excellent choroidal blush.

disappeared (Fig. 1d) Melphalan 3mg and Topotecan $0.3 \mathrm{mg}$ were infused. A follow-up exam one month later showed resolution of the hemorrhage and marked regression of the tumor but ultrasound revealed massive material within the choroid (Fig. 2a) consistent with hemorrhage or choroidal invasion, and the eye was enucleated. Pathological examination revealed massive subretinal hemorrhage (Fig. $\mathbf{2 b}, \mathbf{c})$ with a completely calcified tumor remnant (Fig. 2c, insert), without evidence of optic nerve, choroidal or extraocular extension. Because of the pathologic findings and the fact that hemorrhage after intra-arterial chemotherapy in previously untreated eyes is rare, hemoglobin electrophoresis was done and the child was found to have $50 \% \mathrm{HbS}$ (Sickle cell trait). Superselective ophthalmic artery delivery of small doses of chemotherapy at high local concentration ("Chemosurgery") was introduced

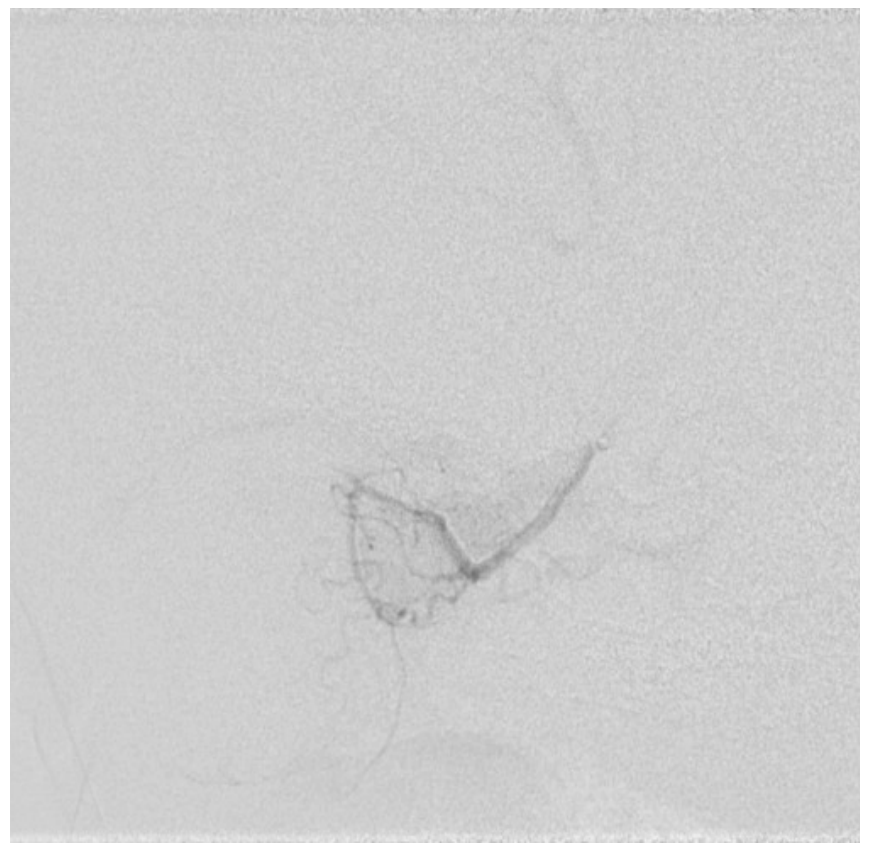

Fig. (1d). Arteriogram during second treatment showing good access in the ophthalmic artery but no choroidal blush.

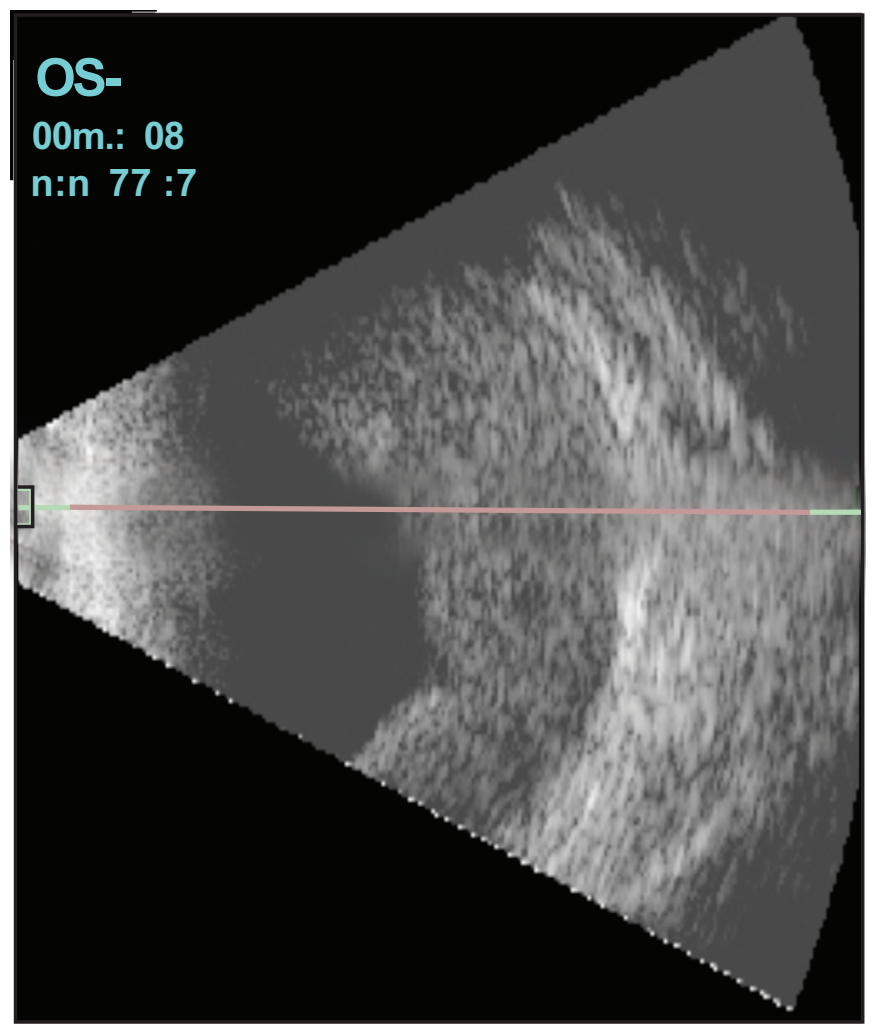

Fig. (2a). B-scan ultrasound after second treatment demonstrating new subretinal material.

by us and has now been performed successfully in 31 countries worldwide [2]. In naïve cases, more than $80 \%$ of eyes can be salvaged with this technique and retinal function is retained or improved the majority of eyes. In our previously reported series, when enucleation was performed following Chemosurgery it was always been for progression of disease and not complications from treatment [3]. In this case 


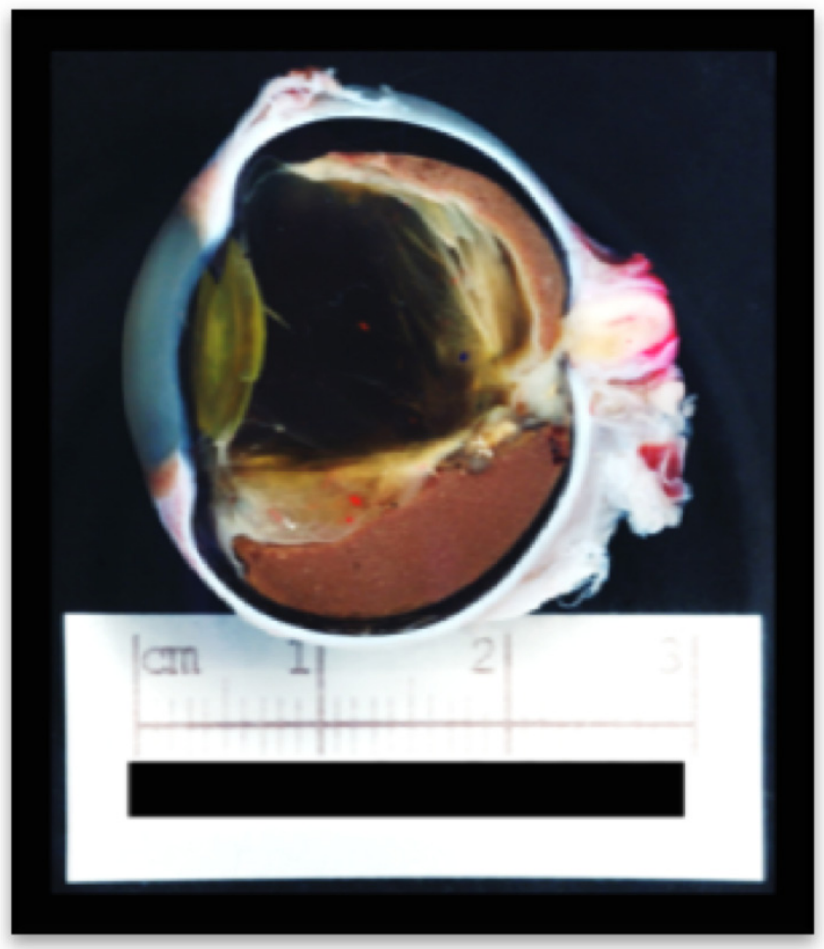

Fig. (2b). Gross of eye enucleated demonstrating subretinal blood.

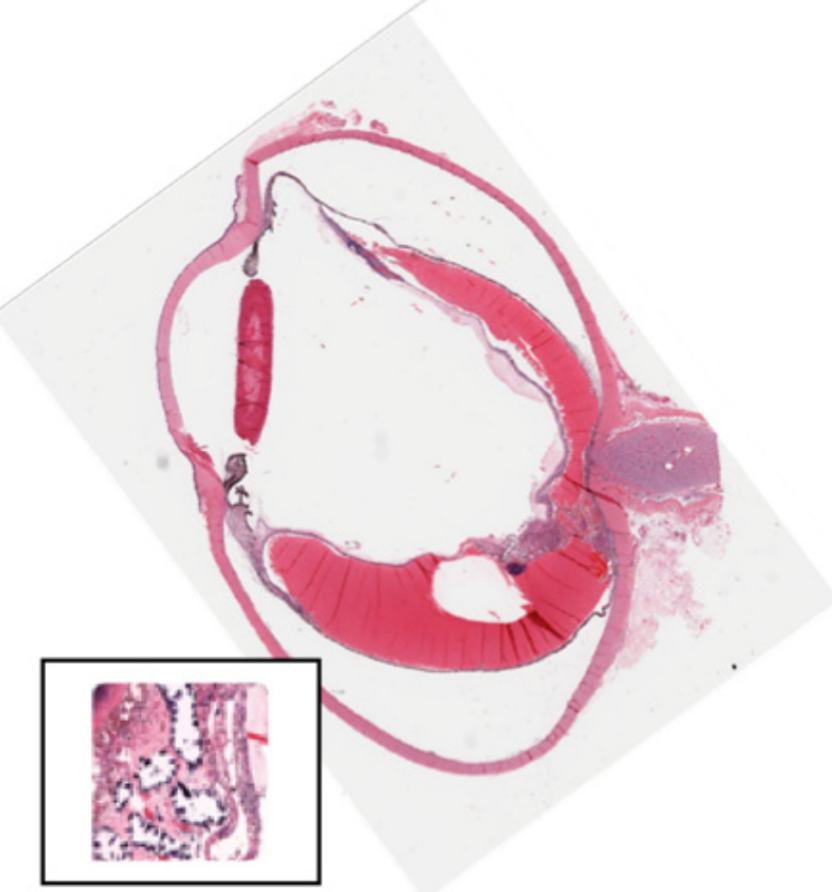

Fig. (2c). Histology of eye after enucleation (insert demonstrates a completely calcified retinoblastoma). There is massive subretinal hemorrhage. There is no viable tumor. enucleation was performed because of the rapid development of subretinal or choroidal masses thought to be consistent with either choroidal invasion or hemorrhage. Systemic and ocular problems from sickle cell disease are well known, but both systemic and ocular complications with sickle trait have also been reported. Strokes, pulmonary emboli during pregnancy and even death from influenza or vigorous exercise have been related to sickle cell trait [4]. Ocular complications include cilio-retinal artery, central retinal artery and central retinal vein occlusion [5]. All of these share lowered oxygen tension and/or dehydration as a precipitating factor. Normally hemoglobin does not polymerize during passage through capillaries because the transit time is short. Patients with sickle cell trait who have longer transit times, especially if oxygen tension is lowered, do transiently sickle, causing local vascular obstruction6. During chemosurgery the small catheter (400-450 microns) within the small ophthalmic artery of babies (500-1,000 microns) may slow blood flow, increase transit time, decreased oxygen tension, and predispose the eye to sickle induced vascular occlusion. The risk of vascular occlusion with intra arterial chemotherapy may be higher in children with sickle cell disease and sickle cell trait and we encourage clinicians to be on the lookout for such associations.

\section{ACKNOWLEDGMENTS}

Financial support for this project was provided in part by The Fund for Ophthalmic Knowledge, Inc. and Alcon Research Institute.

\section{CONFLICTS OF INTEREST}

The authors have no conflicts of interest to report with regard to this manuscript.

\section{REFERENCES}

[1] Hedreville M, Connes P, Romana M. et al. Central retinal vein occlusion in a sickle cell trait carrier after a cycling race Med Sci Sports Exerc 2009; 41: 14-8

[2] Abramson DH, Dunkel IJ, Brodie SE, Kim JW, Gobin YP. A phase I/II study of direct intraarterial (ophthalmic artery) chemotherapy with melphalan for intraocular retinoblastoma; initial results Ophthalmology 2008; 115: 1398-404.

[3] Graeber CP, Gobin YP, Marr BP. et al. Histopathologic findings of eyes enucleated after treatment with chemosurgery for retinoblastoma. Open Ophthalmol J 2011; 18: 1-5.

[4] Conrad WC, Penner R. Sickle-cell trait and central retinal-artery occlusion. Am J Ophthalmol 1967; 63: 465-8.

[5] Lutty GA, Otsuji Y, Taomato M. et al. Mechanism for sickle red blood cell retention in choroid. Curr Eye Res 2002; 25(3): 163-71. 